\title{
ADSORÇÃO DE ÍONS FOSFATO EM ÓXIDO DE NIÓBIO HIDRATADO
}

\author{
Liana Alvares Rodrigues e Maria Lúcia Caetano Pinto da Silva* \\ Departamento de Engenharia Química, Escola de Engenharia de Lorena, Universidade de São Paulo, CP 116, 12600-000 Lorena \\ - SP, Brasil
}

Recebido em 11/6/08; aceito em 9/12/08; publicado na web em 11/5/09

\begin{abstract}
ADSORPTION OF PHOSPHATE IONS ON HYDROUS NIOBIUM OXIDE. The adsorption kinetics of phosphate on $\mathrm{Nb}_{2} \mathrm{O}_{5} \cdot \mathrm{nH}_{2} \mathrm{O}$ was investigated at initial phosphate concentrations $0.25,0.50$ and $1.00 \mathrm{mg} . \mathrm{L}^{-1}$. The kinetic process was described by a pseudo-secondorder rate model very well. The adsorption thermodynamics was carried out at 298, 308, 318, 328 and $338 \mathrm{~K}$. The positive values of both $\Delta \mathrm{H}$ and $\Delta \mathrm{S}$ suggest an endothermic reaction and increase in randomness at the solid-liquid interface during the adsorption. $\Delta \mathrm{G}$ values obtained were negative indicating a spontaneous adsorption process. The Langmuir model described the data better than the Freundlich isotherm model. The effective desorption could be achieved using water at $\mathrm{pH} 12$.
\end{abstract}

Keywords: adsorption of phosphate ions; kinetics models; Langmuir.

\section{INTRODUÇÃO}

A remoção de íons fosfato de efluentes industriais é de grande interesse devido à quantidade de material processado. As indústrias de fertilizantes, detergentes e pigmentos são as que mais produzem efluentes com este ânion originário de seus processos.

Embora seja um nutriente essencial, quando em excesso promove o crescimento de macro e microorganismos aquáticos que se transformam na principal causa da eutrofização dos corpos de água receptores. ${ }^{1-4} \mathrm{O}$ excesso de íons fosfato em efluentes também causa a acidificação e a degradação dos corpos de água, devido à alta demanda biológica de oxigênio, destruindo a vida aquática. ${ }^{5,6}$

Vários processos têm sido desenvolvidos para a remoção de íons fosfato de efluentes, incluindo a precipitação química, a remoção biológica de fósforo, a cristalização, a adsorção e a troca iônica.

O método mais utilizado atualmente é a precipitação química. ${ }^{7,8}$ Contudo, essa técnica tradicional está sujeita a custos elevados, problemas de manipulação do precipitado e neutralização do efluente, além de ser inadequada para a descontaminação de efluentes contendo íons em baixas concentrações. ${ }^{3,9}$ Com isso, têm-se investigado métodos alternativos como, por exemplo, eletrodiálise, osmose reversa, ultrafiltração e adsorção. 3,8

Alguns métodos físicos apresentam-se muito caros, como no caso da osmose reversa e eletrodiálise, ou ineficiente, removendo apenas cerca de $10 \%$ do total de íons fosfato.

O tratamento biológico pode remover mais que $97 \%$ do total de íons fosfato, mas este processo pode ser variável devido às dificuldades operacionais encontradas. ${ }^{1}$

As grandes vantagens da adsorção sobre as outras técnicas são a possibilidade de regeneração dos adsorventes e a aplicação de materiais de baixo custo no tratamento de efluentes. ${ }^{8,10,11}$

Nos últimos anos, vários adsorventes foram desenvolvidos para a remoção de íons fosfato, tais como hidróxidos duplos lamelares, óxidos hidratados, lama vermelha, dolomita, sulfato de alumínio, hidróxidos metálicos, materiais híbridos, goethite e outros tipos de solos. ${ }^{7,9,12-24}$ Dentre estes materiais, os óxidos metálicos são materiais promissores para a remoção desse íon, por possuírem elevada afinidade pelos mesmos. ${ }^{25}$

\footnotetext{
*e-mail: mlcaetano@dequi.eel.usp.br
}

Neste contexto, o óxido de nióbio hidratado é um material promissor para a remoção de íons fosfato de efluentes por apresentar os requisitos de seletividade e estabilidade necessários ao processo de adsorção, bem como ser de fácil preparação. Este óxido metálico hidratado é praticamente insolúvel em água e na maioria dos ácidos, apresenta estrutura rígida, sofrendo pouca dilatação ou compressão quando imerso em solução aquosa, características estas necessárias para um bom desempenho como adsorvente. ${ }^{26}$

O objetivo deste trabalho foi avaliar os parâmetros cinéticos e termodinâmicos da reação de adsorção de íons fosfato em óxido de nióbio hidratado, preparado pelo método da precipitação em solução homogênea (PSH).

\section{PARTE EXPERIMENTAL}

\section{Preparação do óxido de nióbio hidratado via PSH}

O óxido de nióbio (V) hidratado foi preparado pela dissolução de $3 \mathrm{~g}$ de nióbio metálico em mistura ácida de $\mathrm{HNO}_{3}(65 \%) / \mathrm{HF}$ (40\%) na relação 1:3 molar. Depois de todo o material solubilizado, adicionaram-se $50 \mathrm{~mL}$ de água deionizada, $46 \mathrm{~g}$ carbonato de amônio e $20 \mathrm{~g}$ de ácido bórico. A solução foi agitada e aquecida a 85-90 ${ }^{\circ} \mathrm{C}$ para proporcionar a decomposição do precipitante, gerando in situ as hidroxilas necessárias para a formação do óxido de nióbio hidratado. Em seguida o material foi filtrado, lavado com água deionizada até $\mathrm{pH} 7$ e seco em estufa a $50{ }^{\circ} \mathrm{C}$ até massa constante.

\section{Caracterização do óxido de nióbio hidratado}

O material preparado foi caracterizado por difratometria de raios X (DRX), termogravimetria (TG), análise de área superficial específica pelo método BET, microscopia eletrônica de varredura (MEV) e espectrometria de energia dispersiva (EDS).

Os difratogramas foram obtidos em um difratômetro de raios-X, marca Shimadzu modelo XRD-6000, com fonte de radiação $\mathrm{CuK} \alpha$, $30 \mathrm{kV}, 40 \mathrm{~mA}$, varredura 0,05 ( $2 \theta / 5 \mathrm{~s}$ ) para valores de $2 \theta$ entre 10 e $70^{\circ}$.

A análise térmica foi realizada em uma termobalança, marca Shimadzu modelo TG-50, com razão de aquecimento $20^{\circ} \mathrm{C} \mathrm{min}-1 \mathrm{em}$ fluxo de nitrogênio, no intervalo de temperatura de 25 a $900{ }^{\circ} \mathrm{C}$. 
A medida da área superficial específica para o óxido de nióbio (V) hidratado foi realizada em um analisador de adsorção gasosa (gas sorption analizer), Quantachrome Corporation, sob atmosfera de nitrogênio, com fluxo de $30 \mathrm{~mL} \mathrm{~min}{ }^{-1}$. Para a determinação da área superficial específica usou-se o método BET. ${ }^{27}$ As condições de pré-tratamento foram de $50{ }^{\circ} \mathrm{C}$ sob vácuo por $16 \mathrm{~h}$.

As micrografias foram obtidas em um microscópio eletrônico de varredura Leo1450 VP a baixo vácuo acoplado ao espectrômetro de energia dispersiva (EDS).

\section{Adsorção de íons fosfato}

\section{Cinética da adsorção}

Realizou-se a cinética de adsorção de íons fosfato sobre o óxido de nióbio $(\mathrm{V})$ hidratado pelo processo batelada, à temperatura ambiente e sem ajuste de $\mathrm{pH}$. Adicionou-se $0,1 \mathrm{~g}$ do óxido de nióbio hidratado em frascos de polietileno, com $50 \mathrm{~mL}$ de solução de íons fosfato de concentração conhecida, para tempos de agitação de 0-10 h em um banho termostatizado, marca Dubnoff, com agitação orbital. Ao final de cada período as amostras foram filtradas e analisadas pelo método baseado na formação do complexo fosfomolíbdico usando ácido ascórbico como redutor. ${ }^{28}$ Estudaram-se 3 diferentes concentrações: 0,$25 ; 0,50$ e 1,00 mg.L $\mathrm{L}^{-1}$.

\section{Efeito do pH na adsorção}

Estudou-se o efeito do $\mathrm{pH}$ na adsorção de íons fosfato sobre o óxido de nióbio hidratado através do processo batelada à temperatura ambiente, em duplicata. Adicionou-se $0,1 \mathrm{~g}$ de óxido de nióbio hidratado em frascos de polietileno, com $50 \mathrm{~mL}$ de solução de íons fosfato $1,00 \mathrm{mg} \mathrm{L}^{-1}$, alterando os valores de $\mathrm{pH}$ na faixa entre 2 e 9 . Adicionaram-se soluções de $\mathrm{NaOH}$ e $\mathrm{HCl} 0,1 \mathrm{~N}$ para ajuste do $\mathrm{pH}$ da solução. Os frascos foram agitados por tempo pré-determinado em um banho termostatizado, marca Dubnoff, com agitação orbital. Ao final desse período as amostras foram filtradas e analisadas conforme o método proposto por Radojevic e Bashkin. ${ }^{28}$

\section{Estudo de equilíbrio de adsorção}

Realizou-se a adsorção de íons fosfato sobre o óxido de nióbio (V) hidratado em duplicata, pelo processo batelada à temperatura ambiente, com tempo e pH pré-determinados. Adicionou-se $0,1 \mathrm{~g}$ de óxido de nióbio hidratado em frascos de polietileno com $50 \mathrm{~mL}$ de solução de íons fosfato de diferentes concentrações. A faixa de concentração de íon fosfato estudada variou entre 0,10 e $1,00 \mathrm{mg}$ $\mathrm{L}^{-1}$. Após o período de agitação, a solução foi filtrada. Procedeu-se a leitura das soluções conforme o método proposto por Radojevic e Bashkin. $^{28}$

\section{Determinação dos parâmetros termodinâmicos}

Realizou-se o estudo da termodinâmica de adsorção de íons fosfato sobre o óxido de nióbio (V) hidratado pelo processo batelada. Adicionou-se $0,1 \mathrm{~g}$ de óxido de nióbio hidratado em frascos de polietileno, com $50 \mathrm{~mL}$ de solução de íons fosfato $1,00 \mathrm{mg} \mathrm{L}^{-1}$, alterando-se os valores de temperatura do processo entre 298 e $358 \mathrm{~K}$. Os frascos foram agitados por tempo pré-determinado em um banho termostatizado, marca Dubnoff, com agitação orbital. Ao final desse período, as amostras foram filtradas e analisadas conforme o método proposto por Radojevic e Bashkin. ${ }^{28}$

\section{Dessorção}

Para os experimentos de dessorção, o material utilizado na adsorção de $1,00 \mathrm{mg} \mathrm{L}^{-1}$ de íons fosfato foi filtrado, lavado com água deionizada para remoção dos íons não adsorvidos e transferido para frascos de polietileno com $50 \mathrm{~mL}$ de água deionizada. Os testes de dessorção foram realizados em pH 5 e 12; para correção do $\mathrm{pH}$ utilizaram-se soluções de $\mathrm{NaOH}$ e $\mathrm{HCl} 0,1 \mathrm{~N}$. Os frascos foram agitados pelo mesmo tempo utilizado na adsorção. Ao final desse período, as amostras foram filtradas e analisadas conforme o método proposto por Radojevic e Bashkin..$^{28}$

\section{RESULTADOS E DISCUSSÃO}

Em trabalhos realizados anteriormente, ${ }^{26,29}$ observou-se que a presença do ácido fluorídrico impede a hidrólise do fluoreto metálico, impossibilitando a formação do óxido de nióbio hidratado. Sendo assim, adicionou-se ácido bórico para eliminação do excesso de íons fluoreto presentes no sistema, promovendo a formação de complexos boro-flúor, o que permitiu a geração do precipitado. As reações químicas de 1 a 5 descrevem a preparação do material: ${ }^{26}$

$2 \mathrm{Nb}+5 \mathrm{NO}_{3}^{-}+5 \mathrm{H}^{+} \rightarrow \rightarrow \mathrm{Nb}_{2} \mathrm{O}_{5}+5 \mathrm{HNO}_{2}$

$\mathrm{Nb}_{2} \mathrm{O}_{5}+10 \mathrm{HF} \rightarrow 2 \mathrm{H}_{2} \mathrm{NbOF}_{5}+3 \mathrm{H}_{2} \mathrm{O}$

$4 \mathrm{HF}+\mathrm{H}_{3} \mathrm{BO}_{3} \rightarrow 3 \mathrm{H}_{2} \mathrm{O}+\mathrm{H}^{+}+\left[\mathrm{BF}_{4}\right]$

$2 \mathrm{H}_{2} \mathrm{NbOF}_{5}+10 \mathrm{OH}^{-} \rightarrow \mathrm{Nb}_{2} \mathrm{O}_{5}+10 \mathrm{~F}^{-}+7 \mathrm{H}_{2} \mathrm{O}$

$\mathrm{Nb}_{2} \mathrm{O}_{5}+\mathrm{nH}_{2} \mathrm{O} \rightarrow \mathrm{Nb}_{2} \mathrm{O}_{5} \cdot \mathrm{nH}_{2} \mathrm{O}$

As hidroxilas necessárias para a formação do óxido de nióbio hidratado foram geradas pela decomposição, lenta e gradual, do agente precipitante. ${ }^{26,30}$

$\mathrm{O}$ difratograma de raios $\mathrm{X}$ do material preparado (Figura 1) apresentou picos definidos, revelando seu caráter cristalino, como observado na literatura. ${ }^{26,29}$ Este também apresentou pico abaixo de $20^{\circ}$, o que possibilitou a quantificação do espaçamento lamelar do óxido metálico hidratado. Esse pico é decorrente das distâncias entre as lamelas, sendo possível o uso desse material em processos de troca iônica, intercalação e pilarização. ${ }^{31}$ Verificou-se que $\mathrm{o} \mathrm{Nb}_{2} \mathrm{O}_{5} \cdot \mathrm{nH}_{2} \mathrm{O}$ possui espaçamento lamelar de 6,18 Å. Comparou-se o difratograma obtido com o padrão JCPDS. Como não foram encontradas fichas cristalográficas para o óxido de nióbio hidratado, realizou-se um tratamento térmico no material a $550{ }^{\circ} \mathrm{C}$ por $6 \mathrm{~h}$, para eliminação de todo tipo de água existente. $\mathrm{O}$ difratograma de raios $\mathrm{X}$ do material tratado termicamente $\left(\mathrm{Nb}_{2} \mathrm{O}_{5}\right)$ (Figura 2) corresponde à ficha JCPDS $n^{\circ} 28-317$ do óxido de nióbio. Houve eliminação dos picos abaixo de $20^{\circ} \mathrm{C}$ após o tratamento térmico, o que está condizente com a literatura. ${ }^{26,29}$

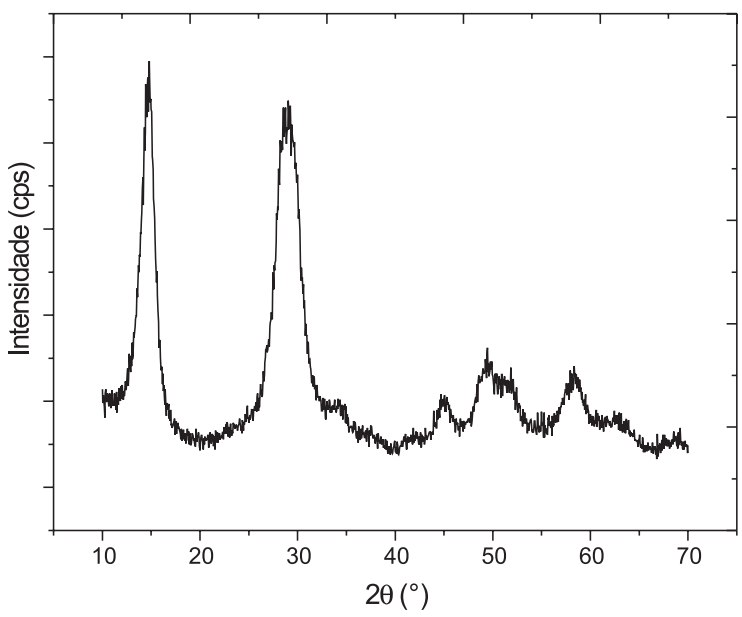

Figura 1. Difratograma de raios $\mathrm{X}$ do $\mathrm{Nb}_{2} \mathrm{O}_{5} \cdot n \mathrm{H}_{2} \mathrm{O}$ 


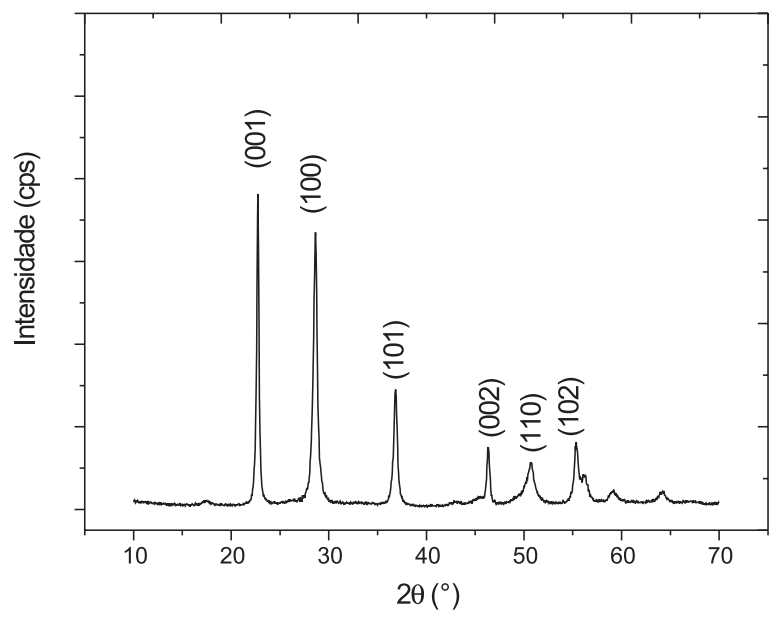

Figura 2. Difratograma de raios $\mathrm{X}$ do $\mathrm{Nb}_{2} \mathrm{O}_{5}$

A termogravimetria foi usada para determinação do grau de hidratação (n) e da estabilidade térmica do material preparado. Esse estudo é importante porque o grau de hidratação corresponde aos sítios ativos de troca iônica, influenciando na capacidade de troca iônica do adsorvente. A curva TG do óxido de nióbio hidratado preparado (Figura 3) apresenta perda de massa em duas etapas distintas na faixa de temperatura de $25-800{ }^{\circ} \mathrm{C}$. Observou-se uma perda de massa de $15,90 \%$ na primeira etapa, entre as temperaturas de 25-200 ${ }^{\circ} \mathrm{C}$, devido à eliminação de águas mais fracamente ligadas à matriz, enquanto que na segunda etapa, entre $200-400^{\circ} \mathrm{C}$, observou-se uma perda de massa de $4,10 \%$, decorrente da condensação dos grupos hidroxilas..$^{26,29}$ A curva DTG mostra que as primeiras águas saem em $115^{\circ} \mathrm{C}$ e as últimas em $277^{\circ} \mathrm{C}$. Com esses dados pode-se calcular o grau de hidratação (n) e, conseqüentemente, sua fórmula estequiométrica $\left(\mathrm{Nb}_{2} \mathrm{O}_{5} \cdot 3,7 \mathrm{H}_{2} \mathrm{O}\right)$.

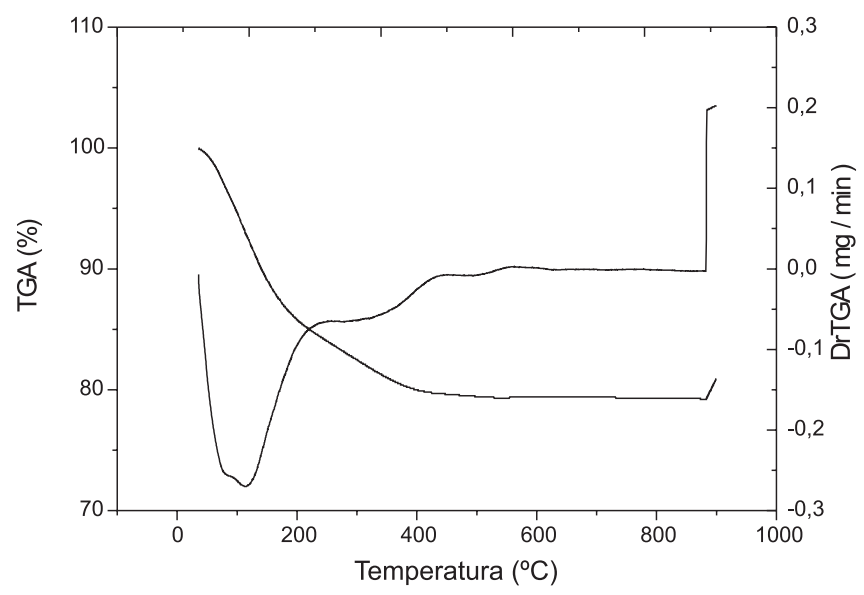

Figura 3. Curva termogravimétrica do $\mathrm{Nb}_{2} \mathrm{O}_{5} \cdot n \mathrm{H}_{2} \mathrm{O}$

A área superficial específica está diretamente relacionada ao sítio ativo da superfície do substrato que, no caso dos óxidos metálicos hidratados, são as hidroxilas. Desta forma, a área superficial também influencia na capacidade de adsorção do material, pois quanto maior a área superficial específica, maior a quantidade de sítios na superfície do material e, conseqüentemente, maior a capacidade de adsorção do mesmo. A área superficial específica do óxido de nióbio hidratado preparado medida pelo método BET é de $0,17 \mathrm{~m}^{2} \mathrm{~g}^{-1}$, valor extremamente baixo quando comparado com outros materiais utilizados na adsorção de íons fosfato (206-404 $\left.\mathrm{m}^{2} \mathrm{~g}^{-1}\right)$. $^{7,10,32,33}$
Pode-se observar através da micrografia apresentada na Figura 4, que o material preparado é pouco poroso e apresenta pequenas partículas esféricas aglomeradas.

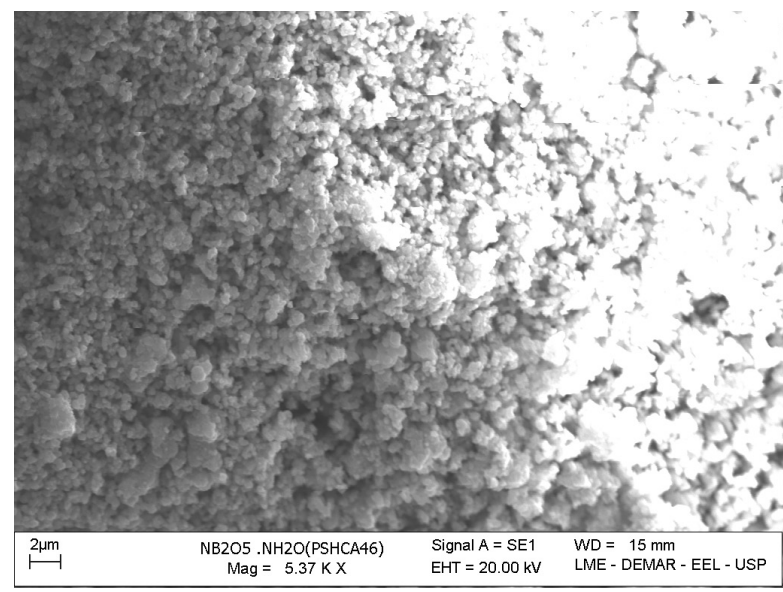

Figura 4. Micrografia do $\mathrm{Nb}_{2} \mathrm{O}_{5} \cdot n \mathrm{H}_{2} \mathrm{O}$ obtidas por $\mathrm{MEV}$

A análise por EDS apresentou $77 \%$ de oxigênio e $23 \%$ de nióbio em porcentagem atômica na composição da matriz. Dessa forma, os dados da análise por EDS confirmam que a matriz preparada é o óxido de nióbio hidratado.

A Figura 5 ilustra a cinética de adsorção de íons fosfato em $\mathrm{Nb}_{2} \mathrm{O}_{5} \cdot \mathrm{nH}_{2} \mathrm{O}$. Observa-se que um aumento na concentração inicial desse íon faz com que seja necessário um tempo de contato maior (30 min) para que se atinja o equilíbrio. Portanto, utilizou-se esse tempo de contato para a realização dos estudos posteriores ( $\mathrm{pH}$, equilíbrio, termodinâmica e dessorção).

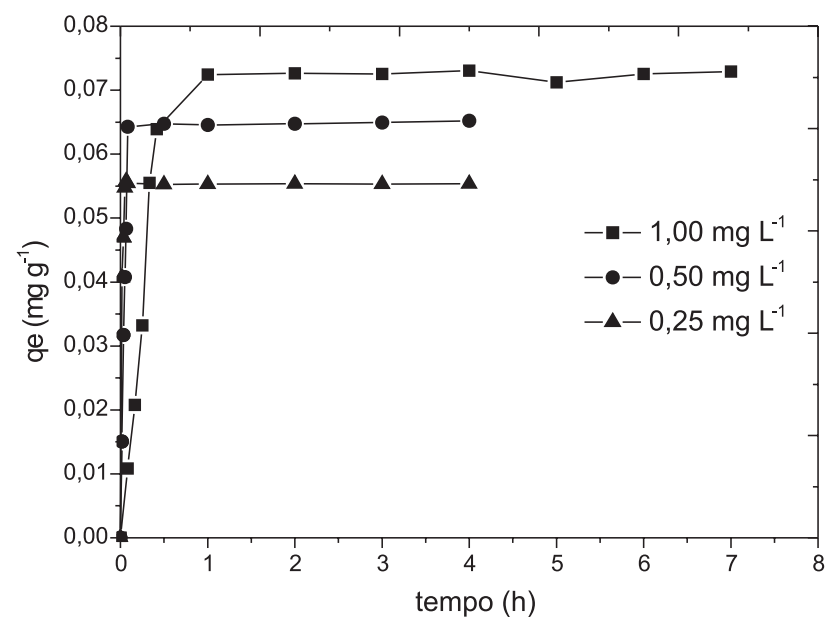

Figura 5. Cinética da adsorção de íons fosfato para o $\mathrm{Nb}_{2} \mathrm{O}_{5} \cdot n \mathrm{H}_{2} \mathrm{O}(\mathrm{pH}=2$ e $T=298 \mathrm{~K}$ )

Estudaram-se os modelos cinéticos de pseudo $1^{\mathrm{a}}$ ordem, de pseudo $2^{\mathrm{a}}$ ordem e de difusão intrapartícula (Figuras de 1S a 3S, Material Suplementar) para examinar o mecanismo que controla o processo de adsorção. ${ }^{8}$ Os estudos cinéticos revelam que a equação de pseudo $2^{\mathrm{a}}$ ordem apresenta o melhor ajuste dos dados experimentais, $\mathrm{r}=1$ (Tabela 1). Para este modelo, a constante diminui com o aumento da concentração inicial. Portanto, a constante é totalmente dependente da concentração inicial de íons fosfato. Um aumento na concentração inicial resulta em um significativo aumento na quantidade de íons fosfato adsorvida no equilíbrio para a equação de pseudo $2^{\mathrm{a}}$ ordem $\left(\mathrm{qe}_{2}\right)$. 
Tabela 1. Parâmetros cinéticos da reação de adsorção de íons fosfato no $\mathrm{Nb}_{2} \mathrm{O}_{5} \cdot \mathrm{nH}_{2} \mathrm{O}$

\begin{tabular}{|c|c|c|c|c|c|c|c|c|}
\hline \multirow{2}{*}{$\begin{array}{l}\text { Co } \\
(\mathrm{mg} / \mathrm{L})\end{array}$} & \multicolumn{3}{|c|}{$1^{\mathrm{a}}$ ordem } & \multicolumn{3}{|c|}{$2^{\mathrm{a}}$ ordem } & \multicolumn{2}{|c|}{ Difusão } \\
\hline & $\mathrm{k}_{1}$ & $\mathrm{qe}_{1}$ & $\mathrm{r}$ & $\mathrm{k}_{2}$ & $\mathrm{qe}_{2}$ & $\mathrm{r}$ & $\mathrm{k}_{\mathrm{in}}$ & $\mathrm{r}$ \\
\hline 1,00 & 6 & 0,13 & 0,90 & 64 & 0,08 & 1,00 & 0,16 & 0,96 \\
\hline 0,50 & 22 & 0,07 & 1,00 & 600 & 0,07 & 1,00 & 0,29 & 0,97 \\
\hline 0,25 & 61 & 0,05 & 0,84 & 3668 & 0,06 & 1,00 & 0,13 & 0,94 \\
\hline
\end{tabular}

Co- Concentração inicial ( $\left.\mathrm{mg} \mathrm{L}^{-1}\right) ; \mathrm{k}_{1}$ - Constante da velocidade pseudo $1^{\mathrm{a}}$ ordem $\left(\mathrm{L} \mathrm{h}^{-1}\right) ; \mathrm{qe}_{1}$ - quantidade de íons fosfato adsorvida no equilíbrio ( $\left.\mathrm{mg} \mathrm{g}^{-1}\right)$; r-Coeficiente de correlação; $\mathrm{k}_{2}$ - Constante da velocidade pseudo $2^{\mathrm{a}}$ ordem $\left(\mathrm{g} \mathrm{mg}^{-1} \mathrm{~h}^{-1}\right) ; \mathrm{qe}_{2}$ - quantidade de íons fosfato adsorvida no equilíbrio para a equação pseudo $2^{\mathrm{a}}$ ordem $\left(\mathrm{mg} \mathrm{g}^{-1}\right) ; \mathrm{k}_{\text {in }}$ - constante da velocidade para difusão dentro da partícula $\left(\mathrm{mg} \mathrm{g}^{-1} \mathrm{~h}^{-0,5}\right)$.

A Figura 3S mostra o gráfico da equação de difusão intrapartícula. Observa-se que o gráfico não é linear para toda faixa de tempo estudada, indicando que mais de um processo afeta a adsorção de íons fosfato em $\mathrm{Nb}_{2} \mathrm{O}_{5} \cdot \mathrm{nH}_{2} \mathrm{O}$. A primeira porção linear está relacionada ao processo de difusão intrapartícula. A segunda porção é atribuída ao estágio final de equilíbrio, no qual a difusão intrapartícula diminui devido à concentração extremamente baixa de íons fosfato em solução. Sendo assim, a constante de velocidade para difusão intrapartícula $\left(\mathrm{k}_{\mathrm{in}}\right)$ foi determinada utilizando-se a primeira porção linear do gráfico. ${ }^{34}$

$\mathrm{O} \mathrm{Nb}_{2} \mathrm{O}_{5} \cdot \mathrm{nH}_{2} \mathrm{O}$ é um óxido metálico anfótero, adsorvendo cátions em meio básico e ânions em meio ácido. A capacidade de troca aniônica é fortemente influenciada pelo $\mathrm{pH}$ da solução, pela espécie iônica do fosfato e pela superfície do adsorvente. A Figura 6 mostra que a quantidade de íons adsorvidos no $\mathrm{Nb}_{2} \mathrm{O}_{5} \cdot \mathrm{nH}_{2} \mathrm{O}$ diminui com o aumento do $\mathrm{pH}$ da solução inicial, de $0,27 \mathrm{mg} \mathrm{g}^{-1} \mathrm{em} \mathrm{pH=2} \mathrm{a} 0,25 \mathrm{mg} \mathrm{g}^{-1} \mathrm{em} \mathrm{pH}=9$. Dependendo do $\mathrm{pH}$ da solução podem existir 3 diferentes espécies iônicas de fosfato: $\mathrm{H}_{2} \mathrm{PO}_{4}^{-}, \mathrm{HPO}_{4}^{-2} \mathrm{ePO}_{4}^{-3}\left(\mathrm{pK}_{1}=2,15, \mathrm{pK}_{2}=7,20\right.$ e $\left.\mathrm{pK}_{3}=12,33\right) .{ }^{15}$ $\mathrm{O}$ aumento na quantidade de fosfato adsorvida com a diminuição do $\mathrm{pH}$ da solução pode ser atribuída ao fato da superfície do $\mathrm{Nb}_{2} \mathrm{O}_{5} \cdot \mathrm{nH}_{2} \mathrm{O}$ estar mais positivamente carregada em meio ácido; com isso, os íons $\mathrm{H}_{2} \mathrm{PO}_{4}$ (espécie predominante em $\mathrm{pH}<4$ ) são mais fortemente atraídos em função do aumento da atração eletrostática. ${ }^{35}$ Analogamente, em meio básico a superfície do $\mathrm{Nb}_{2} \mathrm{O}_{5} \cdot \mathrm{nH}_{2} \mathrm{O}$ está mais negativamente carregada repelindo, assim, os ânions da solução, o que leva a um decréscimo na quantidade adsorvida. Dependência similar do $\mathrm{pH}$ também foi observada na adsorção de íons fosfato em $\mathrm{ZrO}(\mathrm{OH})_{2} .\left(\mathrm{Na}_{2} \mathrm{O}\right)_{0,05} .1,5 \mathrm{H}_{2} \mathrm{O} .{ }^{15}$

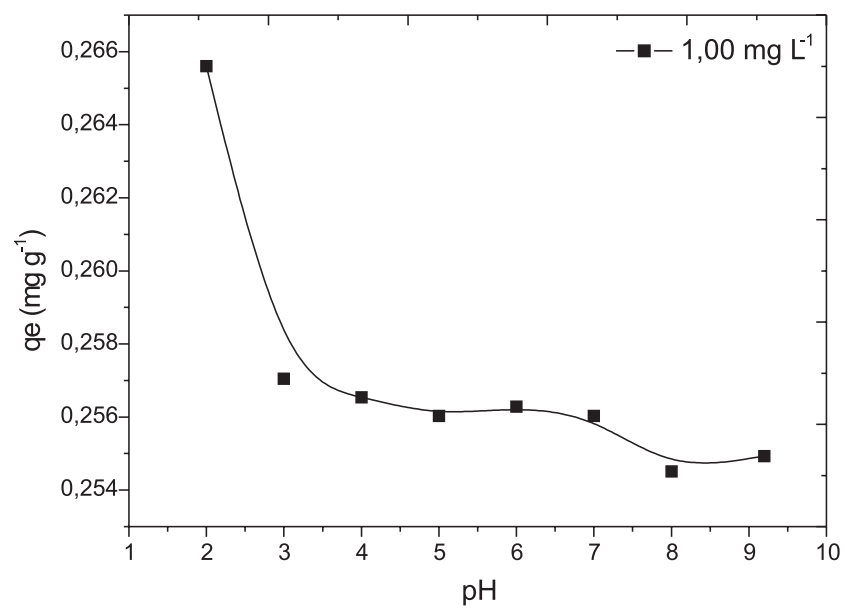

Figura 6. Estudo do efeito do $\mathrm{pH}$ na capacidade de adsorção de íons fosfato do $\mathrm{Nb}_{2} \mathrm{O}_{5} \cdot \mathrm{nH}_{2} \mathrm{O}\left(\mathrm{t}=30 \mathrm{~min}, \mathrm{~T}=298 \mathrm{~K}_{\left.\text {e } \mathrm{C}_{\mathrm{o}}=1,00 \mathrm{mg} \mathrm{L}^{-1}\right)}\right.$
Os dados de adsorção foram ajustados pelos modelos de Langmuir e Freundlich, que são os mais utilizados para o estudo de adsorção de íons fosfato. ${ }^{5,76} \mathrm{O}$ modelo de isoterma de Langmuir considera que o adsorvente possui um número definido de sítios ativos idênticos, onde cada sítio retém apenas uma molécula do adsorvato, que a energia de adsorção não depende da quantidade de material adsorvido e que as espécies adsorvidas não reagem com o meio e nem entre si, sendo a adsorção restrita a uma monocamada. ${ }^{37-40} \mathrm{O}$ modelo de isoterma de Freundlich considera que a adsorção ocorre em multicamadas e é útil para descrever a adsorção em superfícies altamente heterogêneas. ${ }^{33}$

A expressão linear de Langmüir é dada pela Equação 6:

$$
\frac{\mathrm{C}_{\mathrm{e}}}{\mathrm{q}_{\mathrm{e}}}=\frac{1}{\mathrm{Q}_{\mathrm{o}} \mathrm{b}}+\frac{\mathrm{C}_{\mathrm{e}}}{\mathrm{Q}_{\mathrm{o}}}
$$

sendo $\mathrm{C}_{\mathrm{e}}$ a concentração de íons fosfato no equilíbrio $\left(\mathrm{mg} \mathrm{L}^{-1}\right), \mathrm{q}_{\mathrm{e}} \mathrm{a}$ quantidade adsorvida no equilíbrio $\left(\mathrm{mg} \mathrm{g}^{-1}\right), \mathrm{Q}_{\mathrm{o}}$ a constante relacionada com a capacidade de adsorção máxima $\left(\mathrm{mg} \mathrm{g}^{-1}\right)$ e b a constante relacionada com a energia de adsorção $\left(\mathrm{L} \mathrm{mg}^{-1}\right)$. A característica essencial da isoterma pode ser expressa pela constante adimensional chamada parâmetro de equilíbrio $\left(\mathrm{R}_{\mathrm{L}}\right)$, dada pela Equação 7 :

$\mathrm{R}_{\mathrm{L}}=\frac{1}{1+\mathrm{bC}_{\mathrm{o}}}$

sendo $\mathrm{C}_{\mathrm{o}}$ a concentração inicial mais alta $\left(\mathrm{mg} \mathrm{L}^{-1}\right)$ e b a constante de Langmuir. Se $0<\mathrm{R}_{\mathrm{L}}<1$ a adsorção é favorável.

A forma linear de Freündlich é dada pela Equação 8:

$\log q_{e}=\log K_{F}+\frac{1}{n} \log C_{e}$

sendo $\mathrm{q}_{\mathrm{e}}$ a quantidade de íons fosfato adsorvida no equilíbrio (mg $\left.\mathrm{g}^{-1}\right), \mathrm{C}_{\mathrm{e}}$ a concentração na fase líquida no equilíbrio $\left(\mathrm{mg} \mathrm{L}^{-1}\right), \mathrm{K}_{\mathrm{F}} \mathrm{a}$ constante relacionada com a capacidade de adsorção e $1 / \mathrm{n}$ a constante de Freündlich. Se o valor de $1 / n$ é menor que 1 a adsorção é favorável.

As isotermas de Langmuir e de Freundlich do óxido de nióbio hidratado são mostradas nas Figuras 7 e 4S.

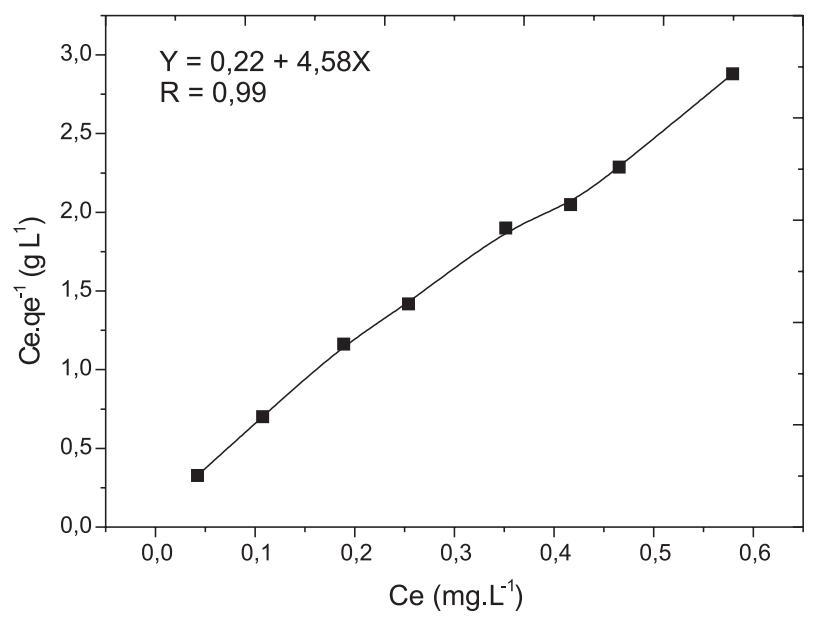

Figura 7. Isoterma de Langmuir para adsorção de íons fosfato no $\mathrm{Nb}_{2} \mathrm{O}_{5}$. $n \mathrm{H}_{2} \mathrm{O}(\mathrm{pH}=2, \mathrm{t}=30$ min e $\mathrm{T}=298 \mathrm{~K})$

As constantes de Langmuir e de Freundlich foram determinadas pela regressão linear das equações linearizadas e estão listadas na Tabela 2. 
Tabela 2. Parâmetros da isoterma de Langmuir para a adsorção de íons fosfato no $\mathrm{Nb}_{2} \mathrm{O}_{5} \cdot \mathrm{nH}_{2} \mathrm{O}$

\begin{tabular}{|c|c|c|c|c|c|c|c|}
\hline \multirow{2}{*}{ Material } & \multicolumn{4}{|c|}{$\begin{array}{l}\text { Parâmetros de } \\
\text { Langmüir }\end{array}$} & \multicolumn{3}{|c|}{$\begin{array}{l}\text { Parâmetros de } \\
\text { Freündlich }\end{array}$} \\
\hline & $\begin{array}{c}\mathrm{Q}_{\mathrm{o}} \\
\left(\mathrm{mg} \mathrm{g}^{-1}\right)\end{array}$ & $\begin{array}{c}\mathrm{b} \\
\left(\mathrm{L} \mathrm{mg}^{-1}\right)\end{array}$ & $\mathrm{R}_{\mathrm{L}}$ & $\mathrm{r}$ & $\mathrm{K}_{\mathrm{f}}$ & $1 / \mathrm{n}$ & $\mathrm{r}$ \\
\hline $\mathrm{Nb}_{2} \mathrm{O}_{5} \cdot \mathrm{nH}_{2} \mathrm{O}$ & 0,22 & 21,30 & 0,08 & 0,99 & 0,22 & 0,18 & 0,97 \\
\hline
\end{tabular}

Os valores dos coeficientes de correlação das retas mostram que o modelo de Langmuir descreve melhor os dados da adsorção e o valor de $\mathrm{R}_{\mathrm{L}}$ indica que a adsorção é um processo favorável na faixa de concentração estudada para o material preparado. Através da constante $\mathrm{Q}_{0}$, observou-se que a capacidade de adsorção máxima do óxido de nióbio hidratado é de $0,22 \mathrm{mg} \mathrm{g}^{-1}$, capacidade 2,5 vezes maior que a do óxido de tungstênio hidratado pesquisado por Rodrigues e Silva. ${ }^{41}$

A distribuição dos íons fosfato entre as fases líquida e sólida é uma medida da posição de equilíbrio no processo de adsorção e o estado deste sistema pode ser definido por um conjunto de propriedades termodinâmicas. ${ }^{42} \mathrm{~A}$ variação de entalpia $\left(\Delta \mathrm{H}^{\circ}\right)$ dada $\mathrm{em} \mathrm{J} \mathrm{mol}^{-1}$, a variação de entropia $\left(\Delta \mathrm{S}^{\mathrm{o}}\right)$ dada em $\mathrm{J} \mathrm{mol}^{-1} \mathrm{~K}^{-1}$ e a variação da energia livre de Gibbs $\left(\Delta \mathrm{G}^{\mathrm{o}}\right)$ dada em J mol ${ }^{-1}$ podem ser determinadas pelas Equações 9 e 10.

$\ln K_{c}=\frac{\Delta S^{\circ}}{R}-\frac{\Delta H^{\circ}}{R T}$

$\Delta \mathrm{G}^{\mathrm{o}}=\Delta \mathrm{H}^{\mathrm{o}}-\mathrm{T} \cdot \Delta \mathrm{S}^{\mathrm{o}}$

sendo $\mathrm{R}$ a constante dos gases $\left(8,314 \mathrm{~J} \mathrm{~mol}^{-1} \mathrm{~K}^{-1}\right)$, T a temperatura absoluta $(\mathrm{K})$ e $\mathrm{K}_{\mathrm{c}}$ a constante de equilíbrio termodinâmica padrão definida por $\mathrm{q}_{\mathrm{e}} / \mathrm{C}_{\mathrm{e}}(\mathrm{mL} / \mathrm{g})$.

Através do gráfico linear de $\ln \mathrm{K}_{\mathrm{c}} v s \mathrm{1} / \mathrm{T}$ (Figura $5 \mathrm{~S}$ ) pode-se estimar os valores de $\Delta \mathrm{H}^{\circ}$ e $\Delta \mathrm{S}^{\circ}$. A equação de reta obtida apresenta coeficiente angular correspondente à $\Delta \mathrm{H}^{\circ} / \mathrm{R}$ e coeficiente linear correspondente à $\Delta \mathrm{S} \% \mathrm{R}^{43}$

Os valores positivo de $\Delta H^{\circ}$ e $\Delta S^{\circ}$ (Tabela 3 ) confirmam a natureza endotérmica da adsorção e o aumento da aleatoriedade da interface sólido/solução durante a adsorção de fosfato em $\mathrm{Nb}_{2} \mathrm{O}_{5} \cdot \mathrm{nH}_{2} \mathrm{O}$. Os valores negativos de $\Delta \mathrm{G}^{\mathrm{o}}$ (Tabela 3) indicam a espontaneidade da reação de adsorção. Com o aumento da temperatura o valor de $\Delta \mathrm{G}^{\mathrm{o}}$ torna-se mais negativo, indicando aumento da eficiência da adsorção com o aumento da temperatura..$^{4,44}$

Tabela 3. Parâmetros termodinâmicos da adsorção de íons fosfato $\mathrm{em} \mathrm{Nb} \mathrm{N}_{5} \cdot \mathrm{nH}_{2} \mathrm{O}$

\begin{tabular}{lcccc}
\hline Material & $\begin{array}{c}\text { Temperatura } \\
(\mathrm{K})\end{array}$ & $\begin{array}{c}\Delta \mathrm{G}^{\mathrm{o}} \\
\left(\mathrm{kJ} \mathrm{mol}^{-1}\right)\end{array}$ & $\begin{array}{c}\Delta \mathrm{H}^{\mathrm{o}} \\
\left(\mathrm{kJ} \mathrm{mol}^{-1}\right)\end{array}$ & $\begin{array}{c}\Delta \mathrm{S}^{\mathrm{o}} \\
\left(\mathrm{J} \mathrm{mol}^{-1} \mathrm{~K}^{-1}\right)\end{array}$ \\
\hline 298 & $-18,55$ & & \\
& 308 & $-19,55$ & & \\
$\mathrm{Nb}_{2} \mathrm{O}_{5} \cdot \mathrm{nH}_{2} \mathrm{O}$ & 318 & $-20,56$ & 11,43 & 100,60 \\
& 328 & $-21,57$ & & \\
& 338 & $-22,57$ & & \\
\hline
\end{tabular}

A dessorção de íons fosfato foi de 90,48 em pH 12 e de $50 \%$ em pH 5. Estes dados indicam que a adsorção é reversível e que os íons fosfato podem ser dessorvidos da superfície do $\mathrm{Nb}_{2} \mathrm{O}_{5} \cdot \mathrm{nH}_{2} \mathrm{O}$ através do ajuste do pH da solução.
Considerando-se o valor de $\Delta \mathrm{H}^{\circ}$ encontrado (menor que $40 \mathrm{~kJ}$ $\mathrm{mol}^{-1}$ ) e a reversibilidade da reação, pode-se afirmar que a adsorção de íons fosfato em $\mathrm{Nb}_{2} \mathrm{O}_{5} \cdot \mathrm{nH}_{2} \mathrm{O}$ é física. ${ }^{44,45}$

\section{CONCLUSÃO}

Os resultados demonstram que o processo de adsorção é influenciado pelo $\mathrm{pH}$ da solução e que o mecanismo cinético segue o modelo de pseudo $2^{\mathrm{a}}$ ordem. A Equação de Langmuir descreve adequadamente os dados de equilíbrio, sendo a capacidade de adsorção máxima de íons fosfato sobre o óxido de nióbio hidratado igual a $0,22 \mathrm{mg} \mathrm{g}^{-1}$. O valor de $\mathrm{R}_{\mathrm{L}}$ indica que a adsorção é um processo favorável na faixa de concentração estudada. A adsorção de íons fosfato em $\mathrm{Nb}_{2} \mathrm{O}_{5} \cdot \mathrm{nH}_{2} \mathrm{O}$ é espontânea e endotérmica $\left(\Delta \mathrm{G}^{\circ}<0\right.$ e $\left.\Delta \mathrm{H}^{\circ}>0\right)$. $\mathrm{O}$ valor positivo de $\Delta \mathrm{S}^{\circ}$ confirma o aumento da aleatoriedade da interface sólido/solução durante a adsorção de fosfato em $\mathrm{Nb}_{2} \mathrm{O}_{5} \cdot \mathrm{nH}_{2} \mathrm{O}$. Os estudos de dessorção indicam que a adsorção é reversível e que os íons fosfato podem ser dessorvidos da superfície do $\mathrm{Nb}_{2} \mathrm{O}_{5} \cdot \mathrm{nH}_{2} \mathrm{O}$ através do ajuste do $\mathrm{pH}$ da solução. $\mathrm{O}$ valor de $\Delta \mathrm{H}^{\circ}$ encontrado (menor que $40 \mathrm{~kJ} \mathrm{~mol}^{-1}$ ) e a reversibilidade da reação indicam que a adsorção de íons fosfato em $\mathrm{Nb}_{2} \mathrm{O}_{5} \cdot \mathrm{nH}_{2} \mathrm{O}$ é física.

\section{MATERIAL SUPLEMENTAR}

As Figuras de $1 \mathrm{~S}$ a $5 \mathrm{~S}$ referentes aos gráficos das equações de pseudo $1^{\mathrm{a}}$ ordem, pseudo $2^{\mathrm{a}}$ ordem, difusão intrapartícula, Freundlich e de $\operatorname{lnKc} v s \mathrm{~T}^{-1}$ estão disponíveis gratuitamente em http://quimicanova.sbq.org.br na forma de arquivo PDF.

\section{AGRADECIMENTOS}

À Fundação de Amparo à Pesquisa do Estado de São Paulo (FAPESP), pela bolsa de mestrado concedida (Processo No 06/05421-0).

\section{REFERÊNCIAS}

1. Karaca, S.; Gürses, A.; Ejder, M.; Açikyildiz, M.; J. Colloid Interface Sci. 2004, 277, 257.

2. Gao, Y.; Mucci, A.; Chem. Geol. 2003, 199, 91.

3. Ozacar, M.; Chemosphere 2003, 51, 321.

4. Namasivayam, C.; Sangeetha, D.; J. Colloid Interface Sci. 2004, 280, 359.

5. Golder, A. K.; Samanta, A. N.; Ray, S.; Sep. Purif. Technol. 2006, 52, 102.

6. Karageorgiou, K.; Paschalis, M.; Anastassakis, G. N.; J. Hazard. Mater. 2007, 139, 447

7. Chubar, N. I.; Kanibolotskyy, V. A.; Strelko, V. V.; Gallios, G. G.; Samanidou, V. F.; Shaposhnikova, T. O.; Milgrandt, V. G.; Zhuravlev, I. Z.; Colloids Surf., A 2005, 255, 55.

8. Spinelli, V. A.; Laranjeira, M. C. M.; Favere, V. T.; Polímeros 2005, 15, 218.

9. Chitrakar, R.; Tezuka, S.; Sonoda, A.; Sakane, K.; Ooi, K.; Hirotsu, T.; J. Colloid Interface Sci. 2005, 290, 45.

10. Ye, H.; Chen, F.; Sheng, Y.; Sheng, G.; Fu, G.; Sep. Purif. Technol. 2006, 50, 283.

11. Özacar, M.; J. Hazard. Mater. 2006, 137, 218.

12. Pradhan, J.; Das, J.; Das, S.; Thakur, R. S.; J. Colloid Interface Sci. 1998, 204, 169.

13. Karaca, S.; Gürses, A.; Ejder, M.; Açikyildiz, M.; J. Hazard. Mater. 2006, 128, 273.

14. Yang, Y.; Zhao, Y. Q.; Babatunde, A. O.; Wang, L.; Ren, Y. X.; Han, Y.; Sep. Purif. Technol. 2006, 51, 193.

15. Chitrakar, R.; Tezuka, S.; Sonoda, A.; Sakane, K.; Ooi, K.; Hirotsu, T.; J. Colloid Interface Sci. 2006, 298, 602.

16. Mulinari, D. R.; Silva, G. L. J. P.; Rodrigues, L. A.; Silva, M. L. C. P.; Cerâmica 2007, 53, 345 . 
17. Zhong, B.; Stanforth, R.; Wu, S.; Chen, J. P.; J. Colloid Interface Sci 2007, 308, 40.

18. Chitrakar, R.; Tezuka, S.; Sonoda, A.; Sakane, K.; Ooi, K.; Hirotsu, T.; J. Colloid Interface Sci. 2006, 297, 426.

19. Luengo, C.; Brigante, M.; Antelo, J.; Avena, M.; J. Colloid Interface Sci. 2006, 300, 511.

20. Falcão, N. P. S.; Silva, J. R. A.; Acta Amaz. 2004, 34, 337.

21. Valladares, G. S.; Pereira, M. G.; Anjos, L. H. C.; Bragantia 2003, 62 , 111.

22. Camargo, O. A.; Castro, O. M.; Vieira, S. R.; Sci. Agric. 1997, 54, 1.

23. Almeida, J. A.; Torrent, J.; Barrón, V.; R. Bras. Ci. Solo 2003, 27, 985.

24. Harvey, O. R.; Rhue, R. D.; J. Colloid Interface Sci. 2008, 322, 384.

25. Antelo, J.; Arce, F. ; Avena, M.; Fiol, S.; López, R.; Macías, F.; Geoderma 2007, 138, 12.

26. Tagliaferro, G. V.; Silva, G. L. J. P.; Silva, M. L. C. P.; Quim. Nova 2005, $28,250$.

27. Brunauer, S., Emmett, P. H.; Teller, E.; J. Am. Chem. Soc. 1938, 60, 309.

28. Radojevic, M.; Bashkin, V. N.; Pratical Enviromental Analysis, MPG Books Ltd: Cornwal, 1999.

29. Peixoto, A. L. C.; Silva, G. L. J. P.; Silva, M. L. C. P.; Cerâmica 2006, $52,249$.

30. Silva, G. L. J. P.; Silva, M. L. C. P.; Caetano, T.; Mater. Res. 2002, 5, 149.

31. Prakash, A. S.; Kamath, P. V.; Hegde, M. S.; Mater. Res. Bull. 2000, 35, 2189.
32. Xiong, J.; He, Z.; Mahmood, Q.; Liu, D.; Yang, X.; Islam, E.; J. Hazard. Mater. 2008, 152, 211.

33. Deliyanni, E. A.; Peleka, E. N.; Lazaridis, N. K.; Sep. Purif. Technol. 2007, 52, 478 .

34. Özacar, M.; Sengil, I. A.; Türkmenler, H.; Chem. Eng. J. 2008, 143, 42.

35. Biswas, B. K.; Inoue, K.; Ghimire, K. N.; Ohta, S.; Harada, H.; Ohto, K.; Kawakita; H.; J. Colloid Interface Sci. 2007, 312, 214.

36. Oguz, E.; J. Colloid Interface Sci. 2005, 281, 62.

37. Sodré, F. F.; Lenzi, E.; Costa, A. C. S.; Quim. Nova 2001, 24, 324

38. Gomri, S.; Seguin, J.-L.; Guerin, J.; Aguir, A.; Sens. Actuators, B 2006, $114,451$.

39. Limousin, G.; Gaudet, J.-P.; Charlet, L.; Szenknect, S.; Barthès, V.; Krimissa, M.; Appl. Geochem. 2007, 22, 249.

40. Ortiz, N.; Susca, C.; Oliveira, K. M. R.; Bressiani, J. C.; Cerâmica 2003, 49, 216.

41. Rodrigues, L. A.; Silva, M. L. C. P.; Cerâmica 2008, 54, 92.

42. Soares, M. R.; Alleoni, L. R. F.; Casagrande, J. C.; Quim. Nova 2005, 28,1014 .

43. Hameed, B. H.; Ahmad, A. A.; Aziz, N.; Chem. Eng. J. 2007, 133, 195.

44. Unuabonah, E. I.; Adebowale, K. O.; Olu-Owolabi, B. I.; J. Hazard. Mater. 2007, 144, 386.

45. Debnath, S.; Ghosh, U. C.; J. Chem. Thermodynamics 2008, 40, 67. 